\title{
On Planetary-Mass Compact Objects as Dark Matter
}

\author{
Erik Zackrisson, Carina Persson, and Nils Bergvall \\ Uppsala Astronomical Observatory, Box 515, SE 75120 Uppsala, \\ Sweden
}

\begin{abstract}
We assess to what extent the claimed signature of planetarymass compact objects in the long-term optical light curves of quasars really can be explained by the dark matter populations allowed in the currently favoured $\Omega_{\mathrm{M}}=0.3, \Omega_{\Lambda}=0.7$ cosmology. Revised upper limits on the cosmological density of planetary-mass compact objects inferred from the statistics of quasar light curves and the equivalent widths of quasar emission lines are also presented.
\end{abstract}

\section{Introduction}

It has been suggested that a substantial fraction of the dark matter of the universe could exist in the form of planetary-mass compact objects. Possible evidence for such objects come in the form of short-term fluctuations in the different images of Q0957+561 (Schild 1996) and the long-term optical variability of singly-imaged quasars (Hawkins 2001). Although the MACHO/EROS results impose severe upper limits on the mass fraction that such objects can contribute to the dark halo of the Milky Way, these constraints may be circumvented by either diffuse (Walker \& Wardle 1999) or highly clustered lenses (Gibson \& Schild 1999). At the present time, the strongest upper limits on the cosmological density of compact objects, $\Omega_{\text {compact }}$, come from predicted effects of quasar microlensing. Here, we will use the two most powerful such methods to investigate how large $\Omega_{\text {compact }}$ is allowed to be for objects in the mass range $10^{-5}-1 M_{\odot}$, fully taking the current uncertainties in the typical size of the optical continuum-emitting region of quasars, $R_{\mathrm{QSO}}$, into account. We also assess to what extent the long-term optical variability of quasars really can be attributed to microlensing by planetary-mass objects.

\section{Upper Limits on the Cosmological Density of Compact Objects}

Large populations of compact objects are predicted to cause microlensing variations in optical light curves larger than those actually observed (Schneider 1993; Zackrisson \& Bergvall 2003) and to produce a fraction of quasars with very small emission line equivalent widths. In Figure 1, we display the upper limits on $\Omega_{\text {compact }}$ inferred from these two methods separately. The equivalent width method was first implemented by Dalcanton et al. (1994), but has here been generalized to the $\Omega_{\mathrm{M}}=0.3, \Omega_{\Lambda}=0.7$ cosmology and the full range of $R_{\mathrm{QSO}}$ currently allowed. Adopting the common assumption of $R_{\mathrm{QSO}} \leq 10^{13} \mathrm{~m}$, we 

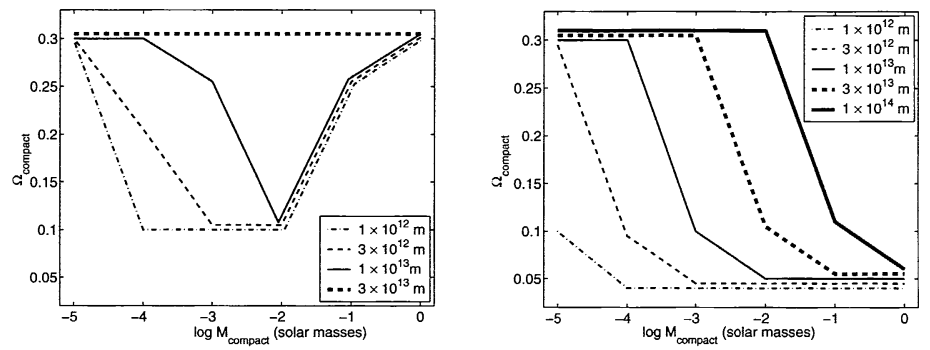

Figure 1. Upper limits on $\Omega_{\text {compact }}$ (at $95 \%$ confidence) inferred from the light curve (left) and emission line equivalent width method (right). The various lines correspond to different assumptions regarding the size of $R_{\mathrm{QSO}}$.

infer $\Omega_{\text {compact }} \leq 0.05$ for $10^{-2}-1 M_{\odot}$ objects and $\Omega_{\text {compact }} \leq 0.1$ for objects at $10^{-3} M_{\odot}$. If instead $R_{\mathrm{QSO}} \geq 3 \times 10^{13} \mathrm{~m}$, planetary-mass $\left(\leq 10^{-3} M_{\odot}\right)$ objects can however constitute essentially all the dark matter. Hence, a dominant dark matter population in this mass range cannot be ruled out at the present time. This conclusion should also apply to the recent study by Wiegert (2003), where the equivalent width method was applied to the quasars from the early data release of the SDSS, but where only $R_{\mathrm{QSO}} \approx 10^{13} \mathrm{~m}$ was considered.

\section{Can Microlensing Explain the Long-Term Optical Variability of quasars?}

Despite providing a natural explanation for the statistical symmetry, achromaticity and lack of cosmological time dilation in optical quasar light curves (Hawkins 2001), we find that microlensing fails to explain the large amplitudes seen at redshifts $z<1$ (Zackrisson et al. 2003). The claimed signature of microlensing in the long-term variability therefore cannot be due to microlensing alone. This severely complicates the task of using light-curve statistics from quasars which are not multiply-imaged to isolate properties of any planetarymass compact object population which may in fact be present.

\section{References}

Dalcanton, J. J., Canizares, C. R., Granados, A., Steidel, C. C., \& Stocke, J. T. 1994, ApJ, 424, 550

Gibson, C. H., \& Schild, R. E. 1999, astro-ph/9904362

Hawkins, M. R. S. 2001, ApJ, 553, 97

Schild, R. E. 1996, ApJ, 464, 125

Schneider, P. 1993, A\&A, 279, 1

Walker, M., \& Wardle, M. 1999, PASA, 16, 262

Wiegert, C. C. 2003, astro-ph/0307465

Zackrisson, E., \& Bergvall, N. 2003, A\&A, 399, 23

Zackrisson, E., Bergvall, N., Marquart, T., \& Helbig, P. 2003, A\&A, 408, 17 